\title{
A Scientific and Economic View on Collaboration, Balancing vs. Sharing
}

\author{
Masayuki Matsui1,2, Tetsuo Yamada1, Masato Takanokura² \\ ${ }^{1}$ The University of Electro-Communications, Tokyo, Japan \\ ${ }^{2}$ Kanagawa University, Yokohama, Japan \\ Email:matsui@se.uec.ac.jp
}

How to cite this paper: Matsui, M., Yamada, T. and Takanokura, M. (2019) A Scientific and Economic View on Collaboration, Balancing vs. Sharing. Theoretical Economics Letters, 9, 49-59. https://doi.org/10.4236/tel.2019.91005

Received: October 9, 2018

Accepted: January 25, 2019

Published: January 28, 2019

Copyright (c) 2019 by author(s) and Scientific Research Publishing Inc. This work is licensed under the Creative Commons Attribution International License (CC BY 4.0).

http://creativecommons.org/licenses/by/4.0/

(c) (i) Open Access

\begin{abstract}
There is a class of artifacts bodies and their science, also known as $3 \mathrm{M} \&$ I-bodies in 2008, which consist of human, material/machine, monetary and informational components. For multi-body systems, the paper discusses and develops the scientific and economic fundamentals of the Follett-like classification as domination, compromise, integration and sharing in 2-center conflict types (Matsui, 1983). That is, the mathematical view is based on the Venn diagram of sets in mathematics, the physical view is based on the principle of the lever in Archimedes' work, and the economical view is based on the profit (specific gravity) and Matsui's equation. From a systematic view composed of these, it is obtained that the integration pursues the maximization of intersection (compromise) in the two-center, whereas the sharing pursues the minimization of intersection (compromise) in field. When the marginal (maximal) profit is the same, both are pointed out to be similar on the relation of classical Nash's solution and duality; the scientific and economic findings will contribute to the advance of the coming society and its visibility and harmony in collaboration vs. sharing.
\end{abstract}

\section{Keywords}

Artifacts-Body, Collaboration, Sharing vs. Integration, Lever/Gravity Rule, Duality

\section{Introduction}

Our study is related to artifacts bodies, also known as 3M \& I-bodies in 2008 [1], which consist of human, material/machine, monetary and informational components. In enterprises or society, based on our past works [1] [2] [3] [4], this paper develops the lever and the balancing principle of collaboration [5], which 
are the integration vs. sharing problem in 3M \& I-body systems, and discusses the digital collaboration and balancing science of multi-bodies in sharing from a mathematical, physical and economical point of view.

For the classical collaboration in homogeneity, traditional collaboration studies began with the conflict problem of sales (demand) vs. manufacturing (supply) non-cooperation in heterogeneity.

Generally, the marketing section in enterprises is interested in the maximization of sales, and, conversely, the manufacturing section is interested in the minimization of costs. However, the difference between sales and costs is not maximized, if there is no cooperation in the functional organization. This original issue is a gaming approach to the joint policy on order-selection (sales) vs. switch-over (manufacturing) model in a job shop at 1983 [2].

This collaboration model, the so-called two-center problem, was first discussed as a management game model (MGM) in 1999 [4]. Our work on the MGM model was summarized in 2008, and the main topic was also presented in a Japanese paper (2005) in "Diamond Harvard Business Review" and was later recorded in English [1].

Recently, Nof et al. [6] discussed the modern collaboration and published an evolutional book on the ICT-engineering of collaboration from the perspective of e-work, business, and services with robotics. The framework could comprehend many types of process (procedure) modeling in cooperation with $3 \mathrm{M} \&$ I-body artifacts originating in our ICPR paper in 1997 [3].

On the other hand, for multi-body systems, our paper re-reviews and develops the Follett-like classification [7] in terms of domination, compromise, integration and sharing in conflict types. The world of economics is now paradigm-changing from ownership (material) to usership (thing), and the subject of collaboration will then transfer from the class of domination/compromise (bottleneck/conflict) to integration/sharing (unification/harmony) toward digitalization.

Especially, the integration pursues the maximization of intersection (compromise) in the two-center, whereas the sharing pursues the minimization of intersection (compromise) in field. When the marginal (maximal) profit is the same, both are found to be similar on the relation of classical Nash's solution and duality.

Also, our original type "invisible collaboration in SCM [8] [9] is similar to A. Smith's economics [10] under demand speed (sharing). This principle of $d$-balancing, in which $d$ means the invisible hand of input type, might be similar to the nonlocality of Quantum Mechanics in physics (spacetime).

The paper discusses and develops the scientific and economic fundamentals of the Follett-like classification as domination, compromise, integration and sharing in 2-center conflict types (Matsui, 1983) [2].

Organizations of this paper as follows. Section 2 addresses collaboration vs. balancing issues, and Section 3 identifies new views of collaboration science. Af- 
ter that, Section 4 discusses coming views of collaboration science. Finally, conclusions and future studies are developed in Section 5.

\section{Collaboration vs. Balancing Issues}

\subsection{Traditional Problem at Business}

For the classical collaboration in homogeneity, traditional collaboration studies began with the conflict problem of sales (demand) vs. manufacturing (supply) non-cooperation in heterogeneity. This original issue is a gaming approach to the joint policy on order-selection (sales) vs. switch-over (manufacturing) model in a job shop at 1983 [2]. Do business and manufacturing really coexist in your company? How can you attempt to measure the effective coexistence but not the conflict?

The conflict in these two functions is a classic, unresolved and heterogeneous problem. This subject is presented as a class of the so-called two-center and $d$-balancing problems by demand speed (d) [1] [5], found in the traditional scheme. From past literatures [1] [7], the scheme is seen in traditional management as follows:

In 1933, a business administration researcher named Mary Parker Follet presented a lecture in London [7]. The topic on which she presented remains relevant in the twenty-first Century. She discussed the functional relations between these sections in the form of "Separation of Planning Division," even though about 100 years have passed since Frederic Taylor's scientific management was generated. It is said that Follet was different, even though there was much criticism of his organization theory according to this function. This problem was also discussed about 30 years ago, but those who conducted research on marketing, including Kotler [11], also admitted the importance of correspondence to be able to achieve harmony between sections. At 1993, cooperative issues were summarized by Eliashberg and Steinberg [12].

How should one resolve the conflict between business and manufacturing and how should one achieve collaboration? We are calling this the "Two-Center Problem" [1] [4]. This problem is where Follet also positively admits the meaning of the conflict, and it is necessary to quickly resolve the problem so that management and society maybe developed.

To resolve this conflict, the relationship between sales (A) and production (B) centers should be classified into domination, compromise and integration [1] [7]. The bottleneck type is a special case of a 2-center model, and the sharing type is added as the extended type of VMI (vender-managed inventory) [13].

Also, our original type "the invisible collaboration in SCM is similar to A. Smith's economics under demand speed (sharing)", and the central (ERP) vs. distributed (series/parallel) type of multi-body in heterogeneity is comparatively discussed [1] [14]. Under digital collaboration, our question is which of integration or sharing would be better on our society in nature vs. artifacts? Probably, this issues would be not yet seen at today's literature. 


\subsection{Collaboration by Objectives vs. Motivations}

For 3M \& I-body, the mathematical, physical and economical view are applied and discussed at the following sections. That is, the mathematical view is based on the Venn diagram of sets, the physical view is based on the principle of the lever in Archimedes' work, and the economical view is based on the profit equation.

The respective collaboration issues in heterogeneous problem could be appropriately re-classified by objectives and motivations in Table 1 .

1) Goal-seeking in optimization

This is similar to the area of operations research (OR/MS). The mathematical (OR-like) view on collaboration gives the representative definition and motivation in the integration of body system by goal-seeking.

2) Leverage balancing in physics

For integration, the opposite category is the sharing in space and time (field), because the integration focuses on the maximization of intersection (compromise), whereas the sharing focuses on its minimization in the field including the complement.

The leverage balancing in physics is motivated at the sharing in space by the principle of lever. For example, there is the sharing in the common file (seat) at cloud/block-chain and in the common car at transportation (so-called car sharing).

3) Demand-balancing in economics

The demand-balancing in economics is motivated at the sharing in time, that is, the medium balancing at demand speed, $d$. This $d$-balancing problem is seen on the upper level of the two-level scheme in the hierarchy. This main problem can be broken down into two sub-problems:

$$
F_{i}\left(I_{i}\right)=\bar{\beta}_{l}, i=1,2, \cdots, n
$$

in the respective body of entity $i$ in the Newsboy problem. Matsui's point (medium), $\bar{\beta}_{i}$, is based on the so-called Chameleon's criteria [14].

Currently, the following condition is considered according to the demand speed (cycle time), $d(0<d<1)$, and the exponential service with the mean, $m_{i}$ (supply speed). That is,

$$
G_{i}(d)=1-\exp \left(-d / m_{i}\right)=\bar{\beta}_{i}, i=1,2, \cdots, n
$$

and the demand speed, $d$, is as follows:

$$
\alpha_{1} \bar{\beta}_{1}=\alpha_{2} \bar{\beta}_{2}=\cdots=\alpha_{n} \bar{\beta}_{n}=(W=Z L) .
$$

In $d$-balancing, the following relation is also obtained from (3):

$$
m_{i} \ln \left(1-\bar{\beta}_{i}\right)=m_{j} \ln \left(1-\bar{\beta}_{j}\right), i \neq j .
$$

In particular, for Poisson service, the optimal condition is

$$
F_{i}\left(I_{i}\right)=\sum_{i=1}^{I_{i}} P\left(d ; m_{i}\right)=1-\bar{\beta}_{i}, i=1,2, \cdots, n
$$


where $P(\cdot)$ is a Poisson type distribution.

Table 1. Collaboration of objectives and motivations in heterogeneity.

\begin{tabular}{cccc}
\hline Triple views & Basics & Objectives & Motivations \\
\hline (a) mathematical & set/logics & integration & goal_seeking \\
(b) physical & lever rule & sharing (space) & leverage balancing \\
(c) economical & specific gravity & sharing (time) & demand balancing \\
\hline
\end{tabular}

These relationships can generally be outlined by the pitch diagram in line balancing. From this pitch diagram and Matsui's equation $W=Z L$ [1] [14], the balance equation is

$$
\sum_{i=1}^{I_{i}} M I_{i}=n Z,
$$

and the second balancing principle is

$$
L \sqrt{Z L}<\sum M I_{i}<Z L=W
$$

from (6) and the classic inequality.

From [8] [9], it is noted that the so-called invisible collaboration would correspond to the balancing state for any $d(>0)$, demand speed (invisible hand).

\section{New Views of Collaboration Science}

\subsection{Domination Type}

Since the industrial revolution, there is seen the separation of ownership and management at collaboration (dominance/compromise). Recently, this relationship is changing to transfer from ownership to usership at collaboration (integration/sharing).

The mathematical view is based on a Venn diagram of sets and logics in mathematics, and on a definition in the multi-body collaboration class. The physical view is based on the principle of the lever and on specific gravity (profit vs. cost ratio at profit equation) in physics (Archimedes' work). The economical view is based on the principle of balancing (win-win) in 3M \& I-body science. In these views, one could totally ascertain the principle of balancing in collaboration issues in Matsui's equation [1] [5].

First, let us consider the type of dominance vs. compromise collaboration in the functional organization. These are well known as the traditional types of collaboration in ownership. For the dominance type of bodies (1) (2) that the body $\mathrm{B}$ (A) belongs and follows or not the body A (B), the three aspects of views are systematically seen in Figures 1-3, respectively, from Matsui [1] as follows:

Figure 1 is the alternative notation of intension in mathematics, and Figure 2 is a physical view on the base of lever rule in Figure 1.

In Figure 3, an optimal condition (balancing) is assumed from the classic inequality and Matsui's equation ( $W=Z L)$ [5] as follows:

Hypothesis: 


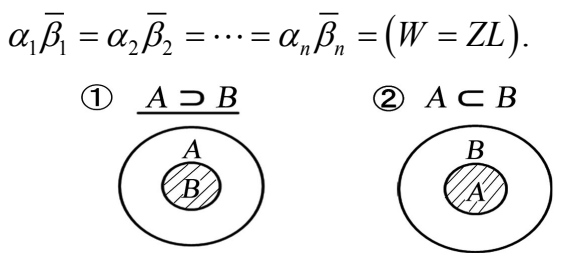

Figure 1. Dominance/occupation ( $A \supset B$ and $A \subset B$ ). Mathematical (set) view.
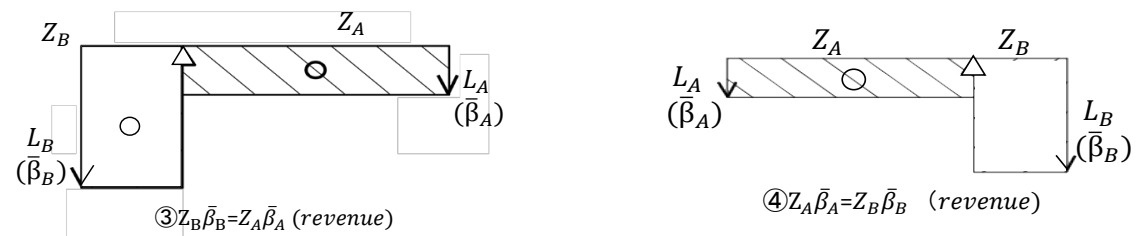

Figure 2. Principle of lever in revenue. Physical (lever) view.

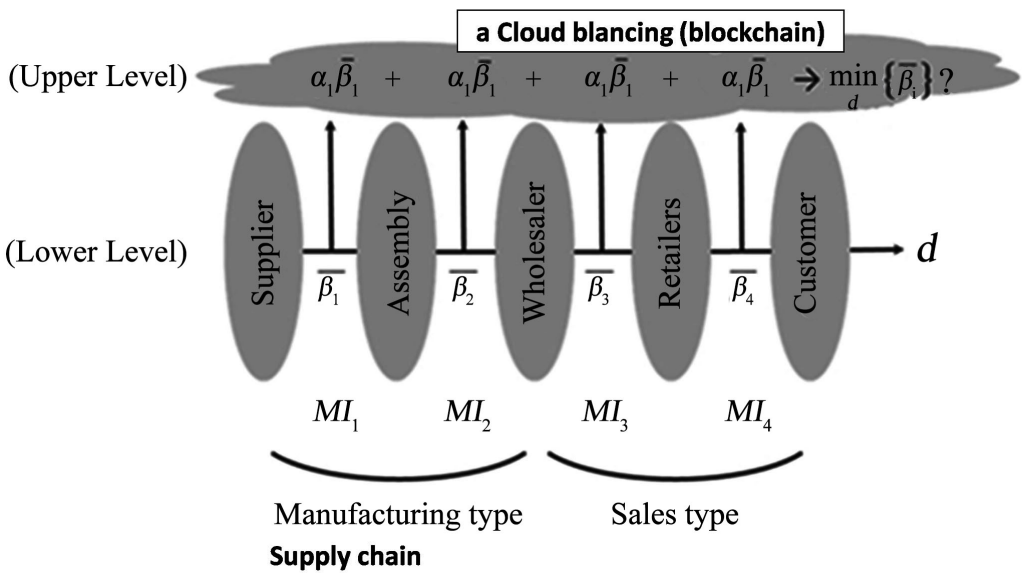

Figure 3. Body-balancing system of supply chain economics in Matsui [14]. Economical (value) view.

In (8), $Z$ and $L$ correspond to $a_{i}$ and $\bar{\beta}_{i}$, respectively, and $W$ means a balancing value at the equilibrium.

\subsection{Compromise Type (A-B)}

At the compromise type of bodies that have common intersection, the three aspects of views are similar to Section 3.1 and are systematically presented as follows:

Figure 4 is the product (5) and sclm (6) in set notation, and Figure 5 is the respective lever rule on the base of specific gravity at Figure 4.

At the trade type of compromise (c) in Figure 6, the win-win strategy in bodies is different to the series vs. parallel type [14]. The former is profit-even and of the SCM (Supply Chain Management) type consisting sales and manufacturing stages in series. Also, the latter is cost-even and of the ERP (Enterprise Resource Planning) type composed of such the multi-functional division as sales 
and production divisions in heterogeneity. Note that both are dualat series vs. parallel type.

(5) $A \cap B$

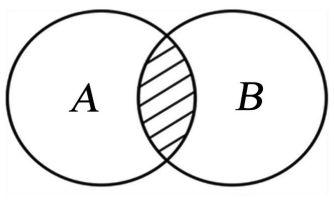

Conflicts vs. sharing (gaming/blocking)
6) $\underline{A \cup B}$

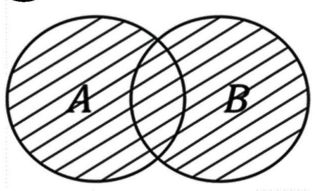

Integration $<$ parallel $>$ II

Collaboration (real?)

Figure 4. Conflicts and integration ( $A \cap B$ and $A \cup B$ ). Mathematical (set) view.
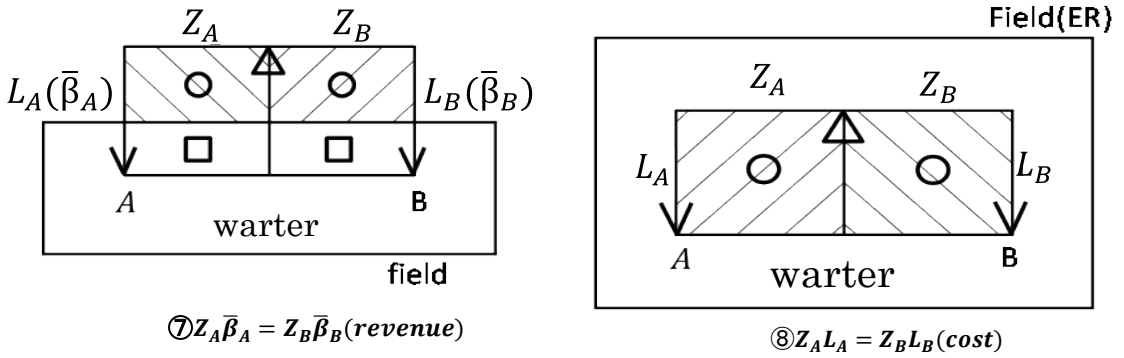

Figure 5. Principle of lever at specific gravity. Physical (lever) view.

(3) $\mathrm{SCM}$

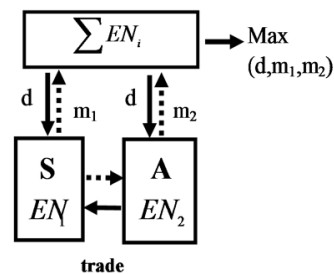

$\rightarrow$ bowl / blocking?

$\left(E \mathrm{C}_{\mathrm{A}} \propto E \mathrm{C}_{\mathrm{B}}\right)$

Series
(4) ERP

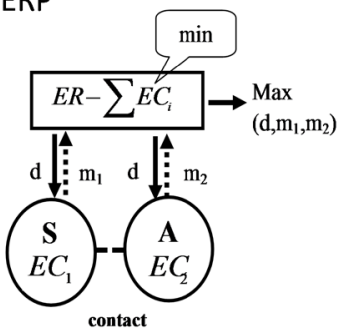

$\mathrm{MEN}: \mathrm{EN}=\mathrm{ER}-\left(E \mathrm{C}_{1}+E \mathrm{C}_{2}\right)$

$\operatorname{Max} \Rightarrow E \mathrm{C}_{1}=E \mathrm{C}_{2}$

Parallel

Figure 6. SCM (series) vs. ERP (parallel) in MGMs. Economical (value) view.

For the class of dominance vs. compromise, it is shown that the compromise is limited or negotiated, and the dominance (occupancy) is vertically maximal at the extent of intersection (conflict). Also, it is noted that the ERP and SCM are dual in parallel vs. series.

\section{Coming Views of Collaboration Science}

\subsection{Integration Type}

Under digitalization and user ship, we would like to ask the next which of integration or sharing is better on our society in nature vs. artifacts? At the integration type of bodies, the three aspects of views are also seen at the sublation of intersection by inclusion as follows: 
By the general definition, it is here noted that there is used the proof by contradiction,

Figure 7 shows the Invisible chain and collaboration: (a), (b) and (c). By the general definition, it is here noted that there is used the proof by contradiction, that is, the contradiction of $\mathrm{A} \wedge \mathrm{B}=\varphi$ (empty) is corresponded to its complement (field). Figure 7(a) relates to the complement of sets A and B. In this type, the following is noted. In the physical view (b), the win-win strategy is the balancing of costs or profits. However, in the economical view (c), it is not the direct sum of profits, but the dual sum or balancing of profits.

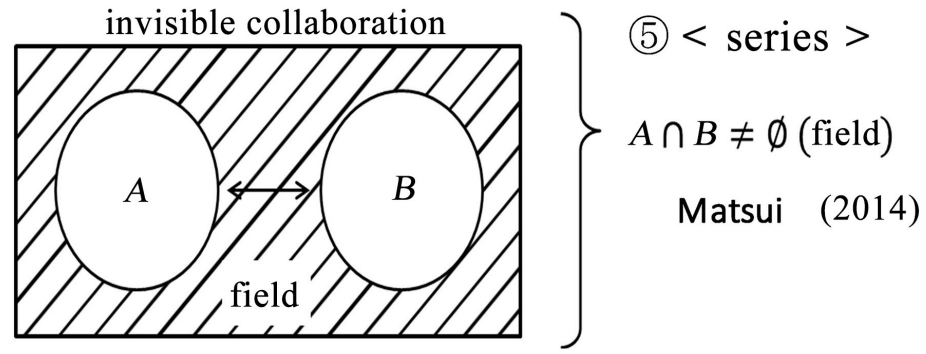

(a)

(8) $Z_{A} L_{A}=Z_{B} L_{B}$ (specific gravify)

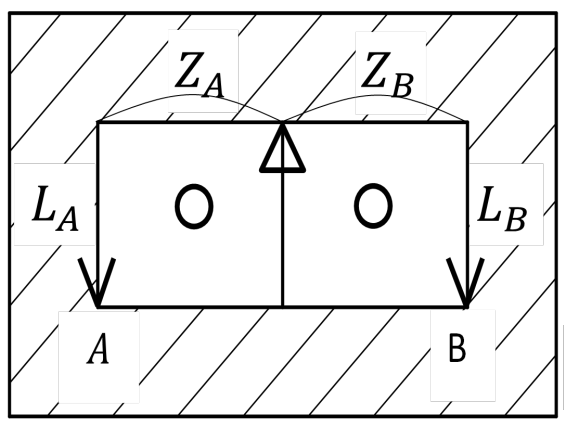

field

(b)

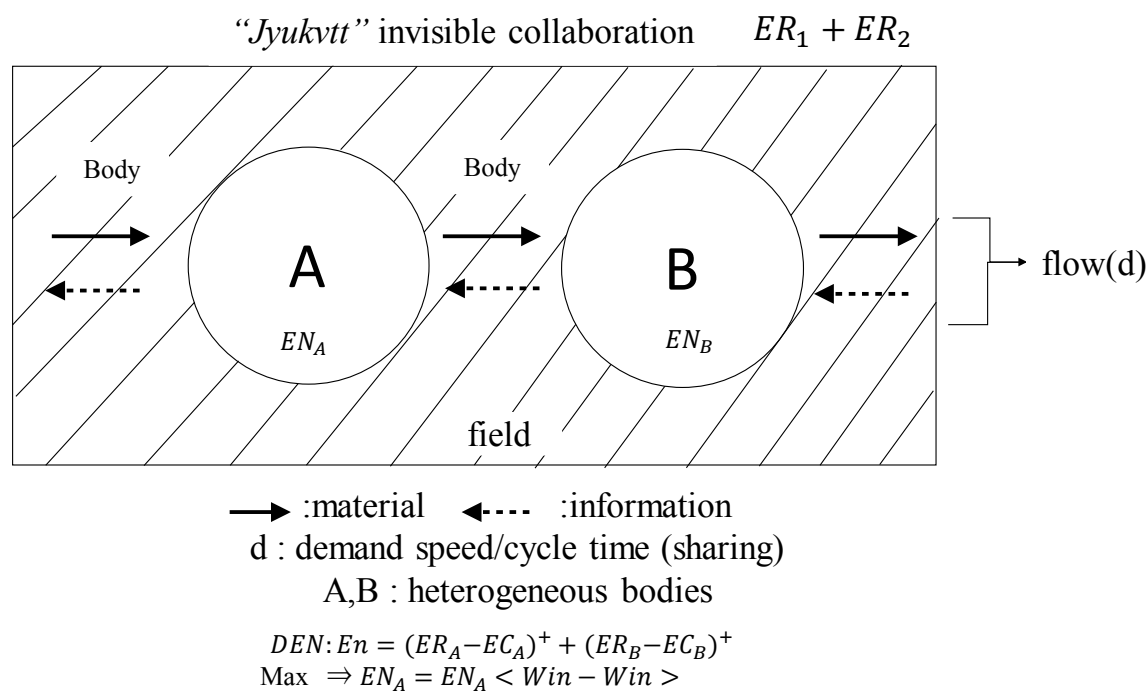

(c) 
Figure 7. Invisible chain and collaboration: (a) Mathematical (set) view; (b) Physical (lever) view; (c) Economical (value) view [5].

\subsection{VMI-Sharing Type}

At the VMI-sharing type of bodies, this type is called the vendor-managed inventory (VMI) at inventory management [13], and many types of sharing have been seen recently in community economics. These views of three aspects are original and are visible as follows:

Figure 8 shows the visible chain and sharing: (a), (b) and (c). Figure 8(a) gives the mathematical notation of sharing type, and shows the sum of slash area in sets A and B. For the class of integration vs. sharing, the followings are noted. At the physical view (b), the weight of the chain (sub-optimal) corresponds to the amount or balancing of costs in bodies. However, at the economical view (c), the strength of the chain (overall optimization) corresponds to the max-product of profits at even-profit.

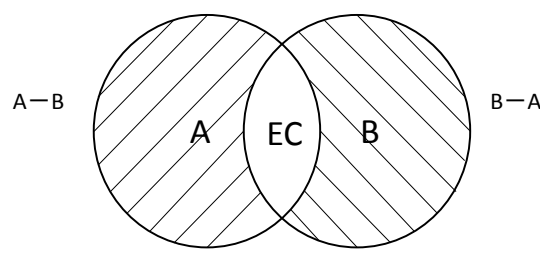

$E C:(A-B)$ or $(B-A)$

$A \Delta B:(A-B) \cup(\mathrm{B}-\mathrm{A})$

(a)

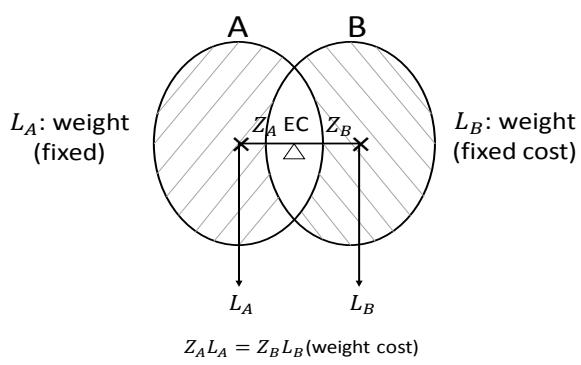

(b)

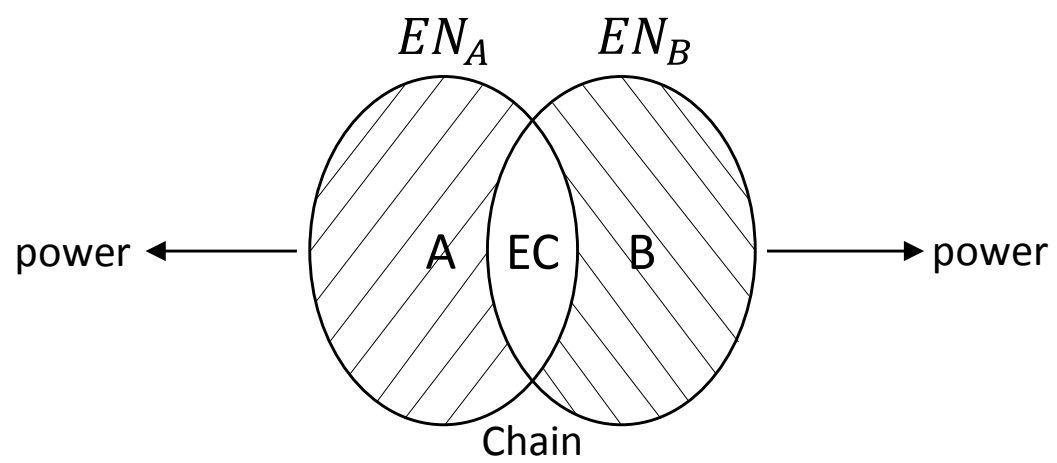

Strength of chain(value chain)

$E N_{A B}=E N_{A} \times E N_{B} \Leftrightarrow \min \left(E N_{A}, E N_{B}\right)$

$\mathrm{EN}$ : profit(specific gravity) EC : operating cost 
(c)

Figure 8. Visible chain and sharing: (a) Mathematical (set) view; (b) Physical (lever) view; (c) Economical (value) view.

Also, from the economical view (c), it is conjectured that the integration is larger than the sharing in different profits. Because, the former is the arithmeticaverage: $E N_{A}+E N_{B} / 2$, and the latter is the geometric mean: $\sqrt{E N_{A} \times E N_{B}}$ in collaboration. Note that both are dual at integration vs. sharing type.

Thus, the maximum of sharing is commutative to the integration (unification), and this means the full sharing in horizontal dominance for different profits. Note that, if each profit of bodies is equal, the sharing is equivalent to the integration. There is seen and critical the classical Nash's solution in duality.

\section{Conclusions}

In this paper, the collaboration and balancing science of multi-bodies were discussed from a mathematical, physical and economical point of view. These three views give a systematic and total view, and the view is consistent with the collaboration vs. balancing principle in [5]. Also, the sublation basis on traditional integration vs. modern sharing under multi-body conflicts is comparatively considered in heterogeneity.

In conclusion, this paper presents the basis of collaboration science toward digitalization, and the integration vs. sharing principle in duality is positioned as the main principal in the $3 \mathrm{M} \& \mathrm{I}$-body system. From now on, the digital collaboration should be transformed from the integration toward sharing issues. The additional subjects are the form utilization and realization toward the analysis and design of artifacts collaboration in connection with the sandwich principal.

From the paper, our study would be valuable to the visibility and harmony of mixture of body (lot) and sharing at digital collaboration, and also, the findings would contribute to the science/engineering design of artifacts (robots) in organizations and societies.

\section{Conflicts of Interest}

The authors declare no conflicts of interest regarding the publication of this paper.

\section{References}

[1] Matsui, M. (2008) The Manufacturing and Service Enterprise with Risks: A Stochastic Management Approach. Springer, New York.

[2] Matsui, M. (1983) A Game-Theoretic Consideration of Order-Selection and Switch-Over Policy. Reprints of Japan Industrial Management Association, Tokyo, 48-49. (In Japanese)

[3] Matsui, M., Ceroni, J. and Nof, S. (1997) A Coordination Consideration of Manufacturing Systems: Job Shop Case. Proceedings of International Conference on Production Research, Osaka, August 1997, 834-837.

[4] Matsui, M. (2002) A Management Game Model: Economic Traffic, Leadtime and 
Pricing Settings. Journal of Japan Industrial Management Association, 53, 1-9.

[5] Matsui, M. (2016) Fundamentals and Principles of Artifacts Science: 3M \& I-Body System. Springer, New York. https://doi.org/10.1007/978-981-10-0473-5

[6] Nof, S.Y., Ceroni, J., Jeong, W. and Moghaddam, M. (2015) Revolutionzing Collaboration through e-Work, w-Business, and e-Service. Springer, New York. https://doi.org/10.1007/978-3-662-45777-1

[7] Follet, M.P. (1949) Freedom \& Co-Ordination: Lecture in Business Organization. Pitman Publishing, London.

[8] Matsui, M. (2010) Division of Work, Stochastic (re-)Balancing and Demand Speed: From Assembly Line toward Demand Chain, Invited Paper. Journal of Japan Industrial Management Association, 60, 324-330.

[9] Matsui, M. (2015) The Invisible Body-Balancing Economics: A Medium Approach. Theoretical Economics Letters, 5, 65-73. https://doi.org/10.4236/tel.2015.51010

[10] Smith, A. (1776) The Wealth of Nations. 1952 Edition, Encyclopedia Britannica.

[11] Kotler, P.T. and Keller, K.L. (1965) Marketing Management. 14th Edition, Pearson.

[12] Eliashberg, J. and Steinberg, R. (1993) Marketing-Production Joint Decision-Making. In: Eli ashberg, J. and Lilien, Q.L., Eds., Marketing, Handbook in OR \& MS, Elsevier, Amsterdam, Vol. 5, Ch. 18.

[13] Yuasa, K. and Matsui, M. (2003) VMI (Vendor Managed Inveutory)-New Business Model toward No-Inventory Management. Nikkan Kogyo Shinbun. (In Japanese)

[14] Matsui, M. (2014) The Manufacturing and Service Enterprise with Risks: II: The Physics and Economics of Management. Springer, New York. https://doi.org/10.1007/978-4-431-54619-1 